\title{
Distance between collapsing matter and apparent horizon in evaporating black holes
}

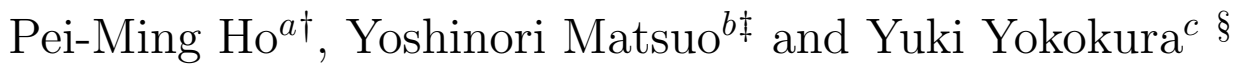 \\ ${ }^{a}$ Department of Physics and Center for Theoretical Physics, \\ National Taiwan University, Taipei 106, Taiwan, R.O.C. \\ ${ }^{b}$ Department of Physics, Osaka University, \\ Toyonaka, Osaka 560-0043, Japan \\ ${ }^{c}$ iTHEMS Program, RIKEN, Wako, Saitama 351-0198, Japan
}

\begin{abstract}
Assuming that the vacuum energy-momentum tensor is not exceptionally large, we consider 4D evaporating black holes with spherical symmetry and evaluate the proper distance $\Delta L$ between the time-like apparent horizon and the surface of the collapsing matter after it has entered the apparent horizon. We show that $\Delta L$ can never be larger than $\mathcal{O}\left(n^{3 / 2} \ell_{p}\right)$ when the black hole is evaporated to $1 / n$ of its initial mass, as long as $n \ll a^{2 / 3} / \ell_{p}^{2 / 3}$ (where $a$ is the Schwarzschild radius and $\ell_{p}$ is the Planck length). For example, the distance between the matter and the apparent horizon must be Planckian at the Page time.
\end{abstract}

\footnotetext{
†e-mail: pmho@phys.ntu.edu.tw

‡e-mail: matsuo@het.phys.sci.osaka-u.ac.jp

$\S$ e-mail: yuki.yokokura@riken.jp
} 


\section{Introduction}

It has been noted [1] that, if the information paradox of black holes is resolved by converting all information of the collapsing matter into the Hawking radiation, there must be highenergy events around the horizon (such as the firewall [2,3]). However, if the collapsing matter is already far inside the horizon, even a firewall around the horizon is still not enough unless there are nonlocal interactions at work.

The question we want to answer in this paper is the following: How far is the collapsing matter under the apparent horizon when the black hole evaporates to a certain fraction of its initial mass, say, one half at the Page time?

For 4D spherically symmetric evaporating black holes, we consider a generic class of vacuum energy-momentum tensor without exceptionally large components. (The conventional model of black holes $[4,6]$ is included.) The back-reaction of the vacuum energy-momentum tensor to the near-horizon geometry is taken into consideration in the semi-classical Einstein equation. The general solution for the near-horizon geometry is consistent with related works on the conventional model (see e.g. Ref. [7]). The light-cone coordinates $(u, v)$ is used so that the causal structure is more manifest.

We prove that, due to a robust exponential form of the redshift factor inside the apparent horizon, the proper distance $\Delta L$ between the apparent horizon and the collapsing matter (after it has fallen inside the apparent horizon) is never larger than $n^{3 / 2} \ell_{p}$ when the black hole is $1 / n$ of its initial mass. This estimate is valid until the black-hole mass is an extremely small fraction $1 / n \sim \mathcal{O}\left(\ell_{p}^{2 / 3} / a^{2 / 3}\right)$ of its initial mass. 1 This conclusion reveals an important feature about the geometry under the apparent horizon. In line with Refs. [9, 10], our result questions the validity of an effective theory with a cutoff scale $\Lambda$ lower than the Planck mass $M_{p}$.

\section{Assumptions}

We start by listing all of our assumptions.

\section{Macroscopic evaporating black hole}

The Schwarzschild radius $a(t) \equiv 2 G_{N} M(t)$ is much larger than the Planck length $\ell_{p} \equiv \sqrt{\hbar G_{N}}$ :

$$
a(t) \gg \ell_{p}
$$

The time-evolution equation for $a(t)$

$$
|\dot{a}| \sim \mathcal{O}\left(\ell_{p}^{2} / a^{2}\right)
$$

\footnotetext{
${ }^{1}$ For the vacuum energy-momentum tensor defined by a 2D massless scalar field [4], the Planck-scale proper distance between apparent horizon and collapsing matter was already argued in Ref. 8.
} 
for the time $t$ of a fiducial observer far from the black hole, so that the time for the black hole to evaporate to a fraction $(<1 / 2)$ of its initial mass is $\mathcal{O}\left(a^{3} / \ell_{p}^{2}\right)$.

\section{Spherical symmetry}

The most general spherically symmetric $4 \mathrm{D}$ metric is

$$
d s^{2}=-C(u, v) d u d v+r^{2}(u, v) d \Omega^{2},
$$

where $u$ and $v$ are the outgoing and ingoing light-cone coordinates, $r(u, v)$ is the areal radius and $d \Omega^{2}$ is the metric of a unit 2-sphere.

\section{Semi-classical Einstein equation}

The semi-classical Einstein equation

$$
G_{\mu \nu}=\kappa\left\langle T_{\mu \nu}\right\rangle
$$

holds since we will focus only on spacetime regions where the curvature is small. Here, $\kappa \equiv 8 \pi G_{N}$ and $\left\langle T_{\mu \nu}\right\rangle$ is the expectation value of the quantum energy-momentum tensor for a given quantum state. Since $\left\langle T_{\mu \nu}\right\rangle$ in vacuum is proportional to the Planck constant $\hbar$, the quantum correction to the Einstein equation is proportional to $\mathcal{O}\left(\ell_{p}^{2}\right)$.

\section{Schwarzschild approximation}

The near-horizon geometry is expected to deviate from the classical solution due to quantum corrections, but it should be smoothly connected to the classical metric at large distances. Within a time $\Delta t \sim \mathcal{O}(a)$ (see eq.(2.2p) in a neighborhood where

$$
r-a \gg \mathcal{O}\left(\frac{\ell_{p}^{2}}{a}\right)
$$

the spacetime geometry is well approximated by a Schwarzschild solution:

$$
C=1-\frac{a}{r}, \quad-\partial_{u} r=\partial_{v} r=\frac{1}{2}\left(1-\frac{a}{r}\right) .
$$

Here we have used the Eddington light-cone coordinates $(u, v)$.

\section{Bounds on energy-momentum tensor}

The vacuum energy-momentum tensor is assumed to satisfy

$$
\left\langle T^{\mu}{ }_{\mu}\right\rangle \lesssim \mathcal{O}\left(\frac{1}{\kappa a^{2}}\right), \quad\left\langle T^{\theta}{ }_{\theta}\right\rangle \lesssim \mathcal{O}\left(\frac{1}{\kappa a^{2}}\right)
$$

in the near-horizon region. These bounds are much weaker than the condition of uneventful horizon for the conventional model $[4-6]$. This quantum effect is important for the apparent horizon. 


\section{Apparent horizon}

We assume that the apparent horizon exists. 22 (See Fig.1 and note its difference from the event horizon, which is irrelevant to our discussion below.) Due to the ingoing negative energy of the vacuum fluctuation, the outer trapping horizon is time-like as it emerges outside the collapsing matter [16 18], [9, 10], [19].

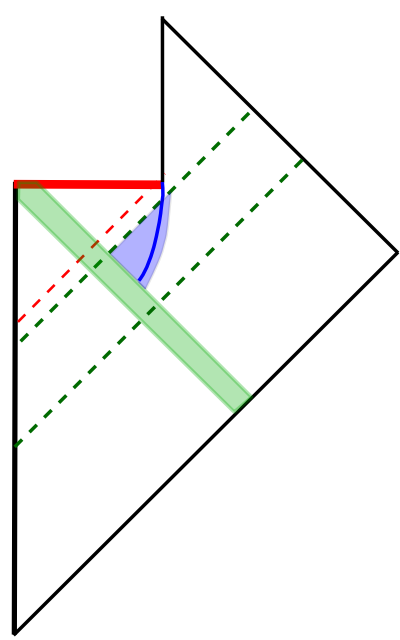

(a)

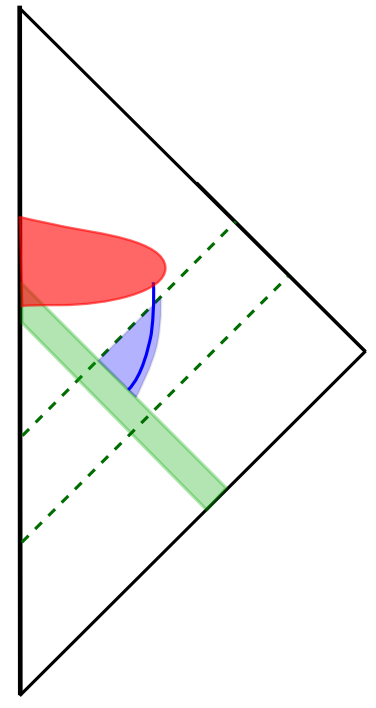

(b)

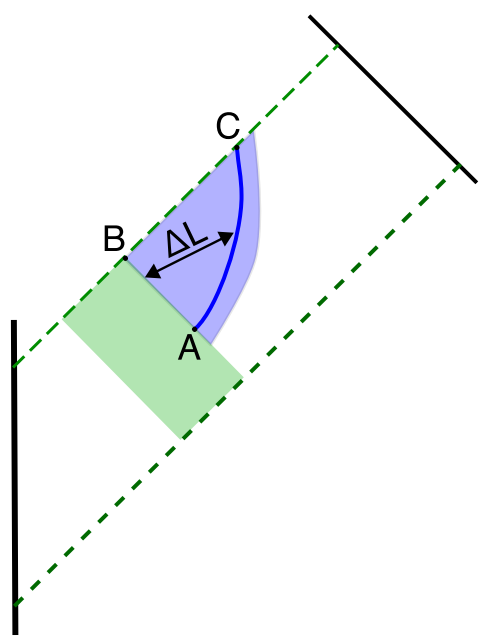

(c)

Figure 1: (a) Penrose diagram for the conventional model. The apparent horizon (blue curve) is time-like outside the collapsing matter (thick green strip) at the speed of light. We focus on the spacetime (between the green dash lines) not long before the apparent horizon emerges, and before the black hole is completely evaporated, from the viewpoint of a distant observer. The event horizon (red dash line) is irrelevant. (b) The singularity at $r=0$ is replaced by a Planckian region (red blob) in the UV theory. The region between the green dash lines remains the same. (c) The region of interest (between the green dash lines) excerpted from (a) and (b). The "near-horizon region" defined in Sec 3.1 is the (blue shaded) region bounded by the following 3 curves: (1) the outer surface of the collapsing matter (thick green strip) from A to B, (2) the outgoing null curve (dashed green line) from B to C, (3) a curve outside but close to the time-like apparent horizon in vacuum from $\mathrm{C}$ to $\mathrm{A}$. We will study the proper length $\Delta L$ of a monotonic radial curve connecting an arbitrary point on the surface of the collapsing matter between A and B and another arbitrary point on the apparent horizon between A and C. An example of such a curve (double arrowhead) is shown.

The quantum correction introduces a difference between the areal radii of the apparent horizon and the Schwarzschild radius as

$$
r\left(u, v_{a h}(u)\right)-a(u) \sim \mathcal{O}\left(\ell_{p}^{2} / a\right) .
$$

\footnotetext{
${ }^{2}$ The appearance of the apparent horizon has been viewed as a definition of black holes 11 13. Note that we are now considering a different situation from Refs. 14, 15].
} 
Here, $a(u)$ is the $u$-dependent Schwarzschild radius which decreases with time as 2.2 , and we use $u_{a h}(v)$ and $v_{a h}(u)$ to denote the $u$ and $v$ coordinates of the apparent horizon for given $v$ and $u$, respectively.

Our assumption on the energy-momentum tensor (2.7) is weaker than (certainly compatible with) what is assumed in the literature for black holes with apparent horizons, for which the energy-momentum tensor would be further constrained by the regularity condition at the apparent horizon $[9,10]$. Here, we aim at the most general energy-momentum tensor for the conclusion of the paper.

\section{Solving Semi-Classical Einstein Equations}

The semi-classical Einstein equation was solved in the near-horizon region in Ref. [8] with the assumption that the vacuum energy-momentum tensor is given by those in Ref. [4]. Here we generalize the result to any vacuum energy-momentum tensor satisfying eq. 2.7.

\subsection{Near-Horizon Region}

We use the phrase "near-horizon region" to refer to the region outside the collapsing matter (with the areal radius $R_{s}(u)$ ) but close to or inside the apparent horizon. See Fig 1 . In view of eqs. 2.5) and (2.7), we choose the outer boundary of the near-horizon region to be

$$
r(u, v)-a(u)=\frac{N \ell_{p}^{2}}{a(u)}
$$

for an arbitrary large number $N$ satisfying $a^{2} / \ell_{p}^{2} \gg N \gg 1$. It follows eqs. 2.6) and 3.1) that the conformal factor in the metric (2.3) around the outer boundary of the near-horizon region is

$$
C(u, v) \sim \mathcal{O}\left(\ell_{p}^{2} / a^{2}\right)
$$

As we will see in the next subsection, this condition is valid anywhere in the near-horizon region.

The trajectory of the outer boundary (3.1) of the near-horizon region (see Fig 2) can be parameterized either by $u$ or by $v$. Using $u$ as the parameter, the $v$-coordinate on the trajectory is denoted by $v_{\text {out }}(u)$. Eq.(3.1) is satisfied with $v=v_{\text {out }}(u)$. Conversely, using $v$ as the parameter, its $u$-coordinate is denoted by $u_{\text {out }}(v)$. Clearly, $u_{\text {out }}$ and $v_{\text {out }}$ are the inverse functions of each other; $u_{\text {out }}\left(v_{\text {out }}(u)\right)=u$ and $v_{\text {out }}\left(u_{\text {out }}(v)\right)=v$.

We focus our attention on a range of $u \in\left(u_{0}, u_{1}\right)$, where $u_{0}$ is the moment when the apparent horizon emerges. We emphasize that in the near-horizon region, the ranges of $u$ and $v$ are both $\sim \mathcal{O}\left(a^{3} / \ell_{p}^{2}\right)$, covering a huge space in terms of the $(u, v)$ coordinates.

The Schwarzschild radius $a(u)$ is monotonically decreasing due to Hawking radiation. 
The maximal and minimal values of $a(u)$ from $u=u_{0}$ to $u_{1}$ are given by

$$
a_{\max }=a\left(u_{0}\right) \quad \text { and } \quad a_{\min }=a\left(u_{1}\right) .
$$

The ratio of the initial and final mass is denoted by

$$
n \equiv \frac{a_{\max }}{a_{\min }}
$$

Note that, for a point $(u, v)$ inside the apparent horizon, where the position of the apparent horizon for a given value of $u$ or $v$ is specified as $\left(u, v_{a h}(u)\right)$ or $\left(u_{a h}(v), v\right)$ (see (2.8)), respectively, we have

$$
v<v_{a h}(u)<v_{\text {out }}(u), \quad u>u_{a h}(v)>u_{\text {out }}(v) .
$$

(See Fig.2.) These inequalities hold because the apparent horizon is timelike, as a result of the negative quantum vacuum energy flow.

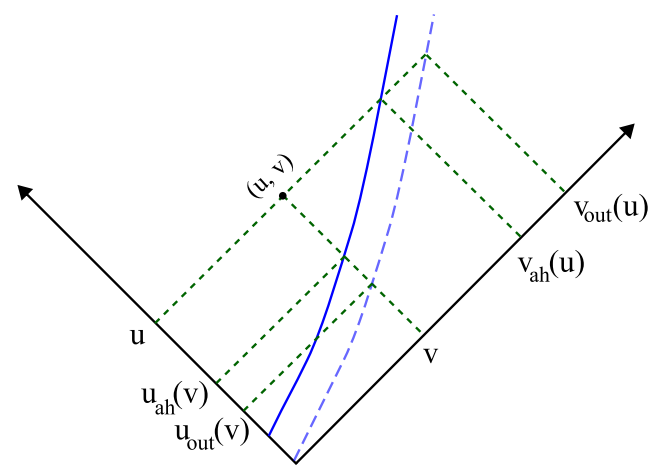

Figure 2: The apparent horizon (solid blue) and the outer boundary of the near-horizon region (dash blue) have their $u, v$ coordinates given by $u_{a h}(v), v_{a h}(u)$ and by $u_{\text {out }}(v), v_{\text {out }}(u)$, respectively. The coordinates $(u, v)$ of a point inside the apparent horizon satisfy eq.(3.5).

\subsection{Solution Of $C(u, v)$}

We solve $C(u, v)$ here from the semi-classical Einstein equation (2.4) in the near-horizon region, with the boundary condition that it agrees with the Schwarzschild approximation.

Consider a particular combination of the semi-classical Einstein equations

$$
G_{\mu}^{\mu}-6 G_{\theta}^{\theta}=\kappa\left(\left\langle T_{\mu}^{\mu}\right\rangle-6\left\langle T_{\theta}^{\theta}\right\rangle\right)
$$


It is equivalent to

$$
\partial_{u} \partial_{v} \Sigma(u, v)=\frac{C(u, v)}{4 r^{2}(u, v)}+\frac{\kappa C(u, v)}{8}\left(\left\langle T_{\mu}^{\mu}\right\rangle-6\left\langle T^{\theta}{ }_{\theta}\right\rangle\right)
$$

where $\Sigma(u, v)$ is defined via

$$
C(u, v) \equiv \frac{e^{\Sigma(u, v)}}{r(u, v)} .
$$

For $r-a \sim \ell_{p}^{2} / a$, the naive order of magnitude of the left-hand side of eq. (3.7) is $\mathcal{O}\left(1 / a^{2}\right)$, and that of the right-hand side is $\mathcal{O}\left(\ell_{p}^{2} / a^{4}\right)$ because of eqs. 2.7) and (3.2). Hence, the leadingorder approximation of eq. 3.7 is

$$
\partial_{u} \partial_{v} \Sigma \simeq 0
$$

It is solved by

$$
\Sigma(u, v) \simeq B(u)+\bar{B}(v)
$$

for arbitrary functions $B(u)$ and $\bar{B}(v)$. To determine $B(u)$ and $\bar{B}(v)$, we impose the boundary condition that $C(u, v)$ matches with the Schwarzschild metric 2.6) around the outer boundary of the near-horizon region. (See Fig 3 .)

According to eq. 3.10$)$, over an infinitesimal variation from $u$ to $(u+d u)$ along a constant$v$ curve, the corresponding change in $\Sigma(u, v)$ is $d u \partial_{u} B(u)$. Since the quantity $d u \partial_{u} \Sigma(u, v)$ is independent of $v$, we take $v=v_{\text {out }}(u)$, where the Schwarzschild solution is a good approximation so that $\Sigma \simeq(v-u) /(2 a)-1+\log (a)$ (see eq. A.6) and Fig.3). We find

$$
\left.d u \partial_{u} B(u) \simeq d u \partial_{u} \Sigma(u, v) \simeq d u \partial_{u} \Sigma_{0}\left(u, v^{\prime}\right)\right|_{v^{\prime}=v_{\text {out }}(u)} \simeq-\frac{d u}{2 a(u)}
$$

Here, we have used A.6 with a time-dependent Schwarzschild radius $a(u)$ and neglected contributions from $\partial_{u} a(u)$ as higher-order terms. Since the procedure above can be repeated for each infinitesimal segment $d u$ for the same $v$, the equation above is immediately solved by

$$
B(u) \simeq B\left(u_{*}\right)-\int_{u_{*}}^{u} \frac{d u^{\prime}}{2 a\left(u^{\prime}\right)}
$$

for an arbitrary reference point $\left(u_{*}, v_{*}\right)$ inside the near-horizon region. The function $a(u)$ can be interpreted as the $u$-dependent Schwarzschild radius for an infinitesimal slice from $u$ to $u+d u$ at the outer boundary of the near-horizon region. (See Fig.3.)

Similarly, we have

$$
\bar{B}(v) \simeq \bar{B}\left(v_{*}\right)+\int_{v_{*}}^{v} \frac{d v^{\prime}}{2 \bar{a}\left(v^{\prime}\right)},
$$

for some function $\bar{a}(v)$, which is the $v$-dependent Schwarzschild radius for an infinitesimal slice from $v$ to $v+d v$ at the outer boundary. (For the static solution, a single slice of the spacetime is enough to determine the Schwarzschild radius. Treating a small slice of the 


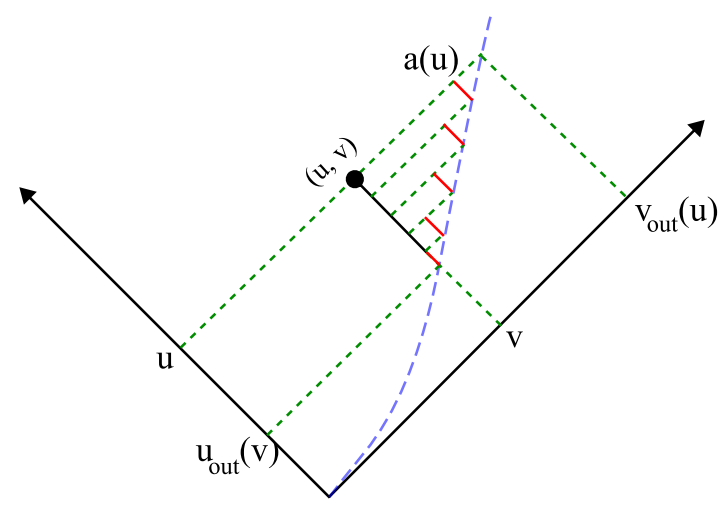

Figure 3: The dash blue curve represents the outer boundary of the near-horizon region. Along the constant- $v$ curve, the $u$-dependence of $\Sigma$ must agree with that of $\Sigma_{0}$ around the boundary (3.1).

dynamical solution as a static one would determine different Schwarzschild radii for slices of fixed $u$ vs. fixed $v ?^{3}$

According to eqs. 3.12 and (3.13), the 0-th order approximation of $\Sigma(u, v)$ is

$$
\Sigma(u, v) \simeq \Sigma\left(u_{*}, v_{*}\right)-\int_{v}^{v_{*}} \frac{d v^{\prime}}{2 \bar{a}\left(v^{\prime}\right)}-\int_{u_{*}}^{u} \frac{d u^{\prime}}{2 a\left(u^{\prime}\right)} .
$$

As a result, we obtain

$$
C(u, v) \simeq C\left(u_{*}, v_{*}\right) \frac{r\left(u_{*}, v_{*}\right)}{r(u, v)} \exp \left[-\int_{u_{*}}^{u} \frac{d u^{\prime}}{2 a\left(u^{\prime}\right)}-\int_{v}^{v_{*}} \frac{d v^{\prime}}{2 \bar{a}\left(v^{\prime}\right)}\right]
$$

Going through the derivation of this solution, one can check that this formula can be applied to any two points $(u, v)$ and $\left(u_{*}, v_{*}\right)$ in the near-horizon region.

We shall adopt the convention of choosing $\left(u_{*}, v_{*}\right)$ to be on the outer boundary of the near-horizon region. To match with the Schwarzschild solution, we have from eq. 3.2.

$$
C\left(u_{*}, v_{*}\right) \sim \mathcal{O}\left(\ell_{p}^{2} / a^{2}\left(u_{*}\right)\right)
$$

In view of eqs. (3.5) and (3.15), $C(u, v)$ becomes exponentially smaller as we go deeper inside the near-horizon region. As we will see below, this is the crucial property of the near-horizon geometry that prevents the distance between the collapsing matter and the apparent horizon from becoming macroscopic until the very late stage of the evaporation.

\footnotetext{
${ }^{3}$ We can evaluate how different the two radii are by constructing the function $r(u, v)$ with a more specific condition of the energy-momentum tensor. See Appendix B of Ref. 20].
} 


\subsection{First-Order Quantum Correction}

Since the large range of both $u$ and $v$ is $\mathcal{O}\left(a^{3} / \ell_{p}^{2}\right)$, the approximation (3.9) might break down after integrating over $u$ or $v$. Here, we show that the approximation is valid over this large range of $u$ and $v$.

The 1st-order quantum correction to $\Sigma(u, v)$ due to the right-hand side of eq.(3.7) can be computed perturbatively as

$$
\begin{aligned}
\Delta \Sigma(u, v) & \simeq \int_{u_{*}}^{u} d u^{\prime} \int_{v_{*}}^{v} d v^{\prime}\left[\frac{1}{4 r^{2}\left(u^{\prime}, v^{\prime}\right)}+\frac{\kappa}{8}\left(\left\langle T_{\mu}^{\mu}\right\rangle+2\left\langle T_{\theta}^{\theta}\right\rangle\right)\right] C\left(u^{\prime}, v^{\prime}\right) \\
& \lesssim\left[\frac{1}{4 r_{\min }^{2}}+\frac{K}{8 a_{\min }^{2}}\right] C\left(u_{*}, v_{*}\right) \frac{r_{\max }}{r_{\min }} \int_{u_{*}}^{u} d u^{\prime} \int_{v_{*}}^{v} d v^{\prime} e^{-\frac{v_{*}-v^{\prime}}{2 a_{\max }}} e^{-\frac{u^{\prime}-u_{*}}{2 a_{\max }}} \\
& \lesssim\left[\frac{a_{\min }^{2}}{r_{\min }^{2}}+\frac{K}{2}\right] \frac{a_{\max }^{2}}{a_{\min }^{2}} \frac{r_{\max }}{r_{\min }} C\left(u_{*}, v_{*}\right),
\end{aligned}
$$

where we have used eq. (3.5) in the last step, and $r_{\max }$ and $r_{\min }$ denote the maximal and minimal values of $r(u, v)$ in the near-horizon region, respectively. We have also used the condition that

$$
\left|\left\langle T^{\mu}{ }_{\mu}\right\rangle+2\left\langle T^{\theta}{ }_{\theta}\right\rangle\right| \leq \frac{K}{\kappa a^{2}}
$$

holds any time (here $K$ is of $\mathcal{O}(1)$ ), according to our assumption (2.7).

The ratios $r_{\max } / r_{\min }$ and $a_{\min } / r_{\min }$ appearing in eq.(3.17) can be estimated without an explicit functional form of $r(u, v)$. First, eqs.2.8 and (3.1) say that both $r\left(u, v_{a h}(u)\right)$ and $r\left(u, v_{\text {out }}(u)\right)$ approximately equal the Schwarzschild radius $a(u)$ up to $\mathcal{O}\left(\ell_{p}^{2} / a\right)$ correction. This is one of the two principles that govern the basic features of the function $r(u, v)$. The other principle is simply the conditions $\partial_{v} r(u, v)<0$ and $\partial_{u} r(u, v)<0$, which hold by definition of the trapped region. Therefore, $r(u, v)$ decreases with $u$ for a fixed $v$. More precisely, $r(u, v)$ on each constant- $v$ curve decreases slower with $u$ for smaller values of $v$ because of the larger red-shift factor for deeper places inside the horizon. Thus, in the nearhorizon region the areal radius becomes the maximum on each constant- $v$ curve at the outer boundary. At the same time, $r(u, v)$ decreases with $v$ for a fixed $u$. The minimum of the areal radius on each constant- $u$ curve is that on the apparent horizon.

With the discussion above, we can draw Fig 4 , as a point-by-point image of Fig.1(c) via the coordinate transformation from $(u, v)$ to $(r, v)$. It should be clear that we can approximate $r_{\max }$ and $r_{\min }$, respectively, as the maximal and minimum Schwarzschild radii $a_{\max }=a\left(u_{0}\right)$ and $a_{\min }=a\left(u_{1}\right)$. Hence we have the relations

$$
\frac{r_{\max }}{r_{\min }} \simeq \frac{a_{\max }}{a_{\min }}, \quad \frac{a_{\min }}{r_{\min }} \simeq 1
$$




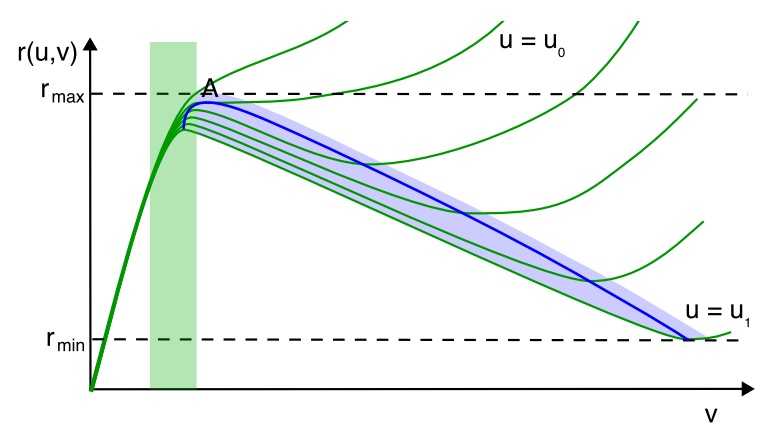

Figure 4: Schematic diagrams of $r(u, v)$ vs $v$. For discrete values of $u$, including $u_{0}$ and $u_{1}, r(u, v)$ is plotted as functions of $v$ (green curves) for given values of $u$. These constant- $u$ curves almost coincide inside the collapsing matter (thick green strip), as well as in the flat spacetime inside the matter shell (on the left of the green strip). Outside the collapsing matter, the local minima of the $r(u, v)$ curves at different $u$ (where $\partial_{v} r(u, v)=0$ ) are located on the apparent horizon $r\left(u_{a h}(v), v\right)$ (blue curve). The near-horizon region is shown as the blue-shaded area.

We thus conclude from eqs. (3.17), (3.16) and (3.19) that

$$
\Delta \Sigma \lesssim \mathcal{O}\left(\frac{n^{3} \ell_{p}^{2}}{a_{*}^{2}}\right)
$$

where $n$ is defined in eq. (3.4).

In the definition of $\Sigma(u, v)$ (3.8), when we write the leading solution 3.15) as $C^{(0)}$, the correction $\Delta \Sigma$ appears as $C=C^{(0)} e^{\Delta \Sigma} \approx C^{(0)}(1+\Delta \Sigma)$. Therefore, the condition for $C$ to be dominated by $C^{(0)}$ is

$$
\frac{C-C^{(0)}}{C^{(0)}} \approx \Delta \Sigma \ll 1
$$

Thus eq. 3.20 means that whenever

$$
n \ll \frac{a^{2 / 3}}{\ell_{p}^{2 / 3}}
$$

holds, the 0 -th order result 3.15 is good.

\section{Distance in Near-Horizon Region}

Define new (Kruskal-like) coordinates (see Fig,50 as

$$
\begin{aligned}
& U \equiv T-X=-2 a_{\max } e^{-\frac{u-u_{*}}{2 a_{\max }}} \in\left(-2 a_{\max }, 0\right), \\
& V \equiv T+X=2 a_{\max } e^{-\frac{v_{*}-v}{2 a_{\max }}} \in\left(0,2 a_{\max }\right)
\end{aligned}
$$


where the range is deduced from the inequalities 3.5 . The proper length along a spacelike curve $\mathcal{C}$ restricted to the $u-v$ plane ${ }^{4}$ is

$$
\begin{aligned}
\Delta L & =\int_{\mathcal{C}} \sqrt{-C(u, v) d u d v} \leq C^{1 / 2}\left(u_{*}, v_{*}\right) \frac{r^{1 / 2}\left(u_{*}, v_{*}\right)}{r^{1 / 2}(u, v)} \int_{\mathcal{C}} \sqrt{-e^{-\frac{u-u_{*}}{2 a_{\max }}} e^{-\frac{v_{*}-v}{2 a_{\max }}} d u d v} \\
& \leq C^{1 / 2}\left(u_{*}, v_{*}\right) \frac{r_{\text {max }}^{1 / 2}}{r_{\text {min }}^{1 / 2}} \int_{\mathcal{C}} \sqrt{-d U d V} \simeq C^{1 / 2}\left(u_{*}, v_{*}\right) \frac{a_{\text {max }}^{1 / 2}}{a_{\text {min }}^{1 / 2}} \int_{\mathcal{C}} \sqrt{d X^{2}-d T^{2}} \\
& \leq C^{1 / 2}\left(u_{*}, v_{*}\right) \frac{a_{\text {max }}^{1 / 2}}{a_{\text {min }}^{1 / 2}} \int_{\mathcal{C}} d X \leq 2 \frac{a_{\text {max }}^{3 / 2}}{a_{\text {min }}^{1 / 2}} C^{1 / 2}\left(u_{*}, v_{*}\right) \sim \mathcal{O}\left(n^{3 / 2} \ell_{p}\right),
\end{aligned}
$$

where we have used eqs. (3.15), (3.16), and (3.19) and the definition $n \equiv a_{\max } / a_{\min }$.

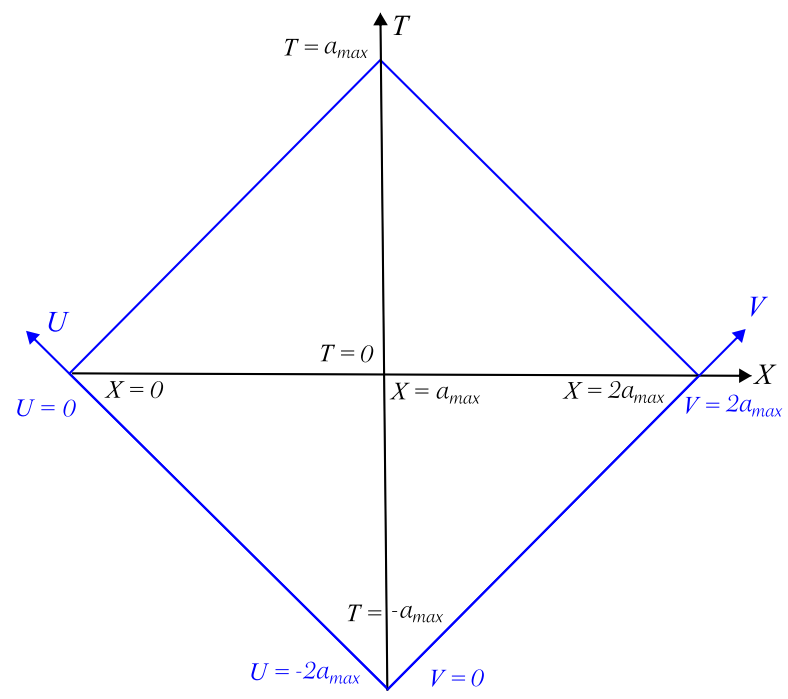

Figure 5: The near-horizon region (the blue-shaded region in Fig 1 is mapped to a subspace of the $U-V$ plane via eqs. (4.1) and (4.2). The distance between any two points in the nearhorizon region is shorter than the distance between their images in this diamond. Corresponding to $U \in\left(-2 a_{\max }, 0\right)$ and $V \in\left(0,2 a_{\max }\right)$, we have $X \in\left(0,2 a_{\max }\right)$ and $T \in\left(-a_{\max }, a_{\max }\right)$. Within this space, the curve with maximal proper distance is the $X$-axis at $T=0$, and the curve with the maximal proper time is the $T$-axis at $X=a_{\max }$.

Via a similar calculation, the proper time for a timelike curve is also at most of the same order of magnitude.

This result means that any proper distance in the radial direction inside the near-horizon region is bounded from above by $n^{3 / 2} \ell_{p}$. In particular, this conclusion applies to the proper distance between the apparent horizon and the surface of the collapsing matter, since both are in the near-horizon region.

\footnotetext{
${ }^{4}$ Each point in the $u-v$ plane represents a 2 -sphere in $4 \mathrm{D}$. The curve $\mathcal{C}$ is restricted to the radial and temporal directions to define the distance between two concentric 2-spheres.
} 
At first glance, this result might seem purely classical because the 0-th order solution $C(u, v)$ (3.15) has been obtained from eq.(3.9), which includes no quantum correction. However, we have arrived at eq. (4.3) by using the inequalities (3.5), which is a consequence of the negative vacuum energy. Hence this result actually relies on the quantum effect.

At the Page time $(n=2)$, the distance between the collapsing matter and the apparent horizon is at most $\ell_{p}$. When $99 \%$ of the black hole is evaporated, the distance is at most 100 times the Planck length.

A peculiar feature of our estimate of the upper bound $\Delta L$ for the proper distance in the near-horizon region is that $\Delta L$ does not depend on both the initial and final masses, but only on their ratio. ${ }^{6}$ As a result, the upper bound $\Delta L$ is the same for black holes that could be dramatically different in size, as long as they have evaporated to the same portion of their initial mass. This is actually a coincidence in the sense that, if we repeat the calculation above for a higher spacetime dimension $D>4$, the estimate $\Delta L$ would no longer have this property. Instead, one has 21

$$
C \simeq C_{*} e^{-(D-3)\left(u-u_{*}+v_{*}-v\right) / 2 a_{*}}, \quad C_{*} \sim \mathcal{O}\left(\ell_{p}^{D-2} / a_{*}^{D-2}\right),
$$

so that the upper bound on the distance is now

$$
\Delta L \sim a_{*} C_{*}^{1 / 2} \sim \ell_{p}^{(D-2) / 2} / a_{*}^{(D-4) / 2}
$$

Hence, $\Delta L$ 's independence of $a_{*}$ (or $M_{*}$ ) is only a coincidence for $4 \mathrm{D}$ black holes.

To summarize, we have shown that, after the collapsing matter enters the apparent horizon and before the black hole's mass is reduced to $1 / n$ of its initial mass, the proper distance $\Delta L$ between the surface of the collapsing matter and the timelike apparent horizon is bounded from above by $\Delta L \leq n^{3 / 2} \ell_{p}$. This bound holds for all paths along which $d \theta=d \phi=0$ as long as $n \ll a^{2 / 3} / \ell_{p}^{2 / 3}$. This result is very interesting because it shows that, from the viewpoint of a low-energy effective theory below the Planckian scale, it is strictly speaking indistinguishable whether the matter has entered the apparent horizon in this regime. Our result is in agreement with Refs. [9, 10], which investigated the implications of the violation of the null energy condition in the near-horizon geometry, and questioned the validity of the low-energy effective theory in the near-horizon regime. Furthermore, we expect to derive from this result further implications to the information paradox [20].

\footnotetext{
${ }^{5}$ Interestingly, another self-consistent model 14 15], in which there is no trapped region, provides a similar result. A collapsing matter is just above the Schwarzschild radius by a Planckian distance. In this sense, the conventional model studied in the present paper might eventually be closely related to such a model.

${ }^{6}$ It is also independent of other details such as when the near-horizon region appears and how long it takes the black hole to evaporate to $1 / n$ of its initial mass.
} 


\section{Acknowledgement}

We thank Hikaru Kawai for valuable discussions and suggestions. P.M.H. thanks iTHEMS at RIKEN, University of Tokyo and Kyoto University for their hospitality during his visits where the major part of this work was done. P.M.H. is supported in part by the Ministry of Science and Technology, R.O.C. and by National Taiwan University. The work of Y.M. is supported in part by JSPS KAKENHI Grants No. JP17H06462 and JP20K03930. Y.Y. is partially supported by Japan Society of Promotion of Science (JSPS), Grants-in-Aid for Scientific Research (KAKENHI) Grants No. 18K13550 and 17H01148. Y.Y. is also partially supported by RIKEN iTHEMS Program.

\section{A Schwarzschild Approximation}

As long as $a \gg \ell_{p}$, the Schwarzschild solution with a time-independent Schwarzschild radius $a$ should be a good approximation within a time scale $\lesssim \mathcal{O}(a)$ at a place well outside the Schwarzschild radius where 7

$$
r-a \gtrsim \frac{N \ell_{p}^{2}}{a}
$$

with a sufficiently large (but finite) $N$ (e.g. $N \sim 10000$ ).

We consider the small neighbourhood outside the apparent horizon 8

$$
r-a \in\left(\frac{N \ell_{p}^{2}}{2 a}, \frac{N \ell_{p}^{2}}{a}\right)
$$

and a period of time $\left[t_{0}, t_{0}+\Delta t\right]$ where $\Delta t \sim \mathcal{O}(a)$. We demand that $N$ is sufficiently large so that the Schwarzschild approximation is good, but not too large $\left(\frac{a^{2}}{\ell_{p}^{2}} \gg N\right)$ so that $(r-a) / a \ll 1$. The metric in the region $(\mathrm{A} .2)$ can be approximated as the usual Schwarzschild metric with a constant radius $a=a\left(t_{0}\right)$ :

$$
d s^{2}=-\left(1-\frac{a}{r(u, v)}\right) d u d v+r^{2}(u, v) d \Omega^{2}
$$

where the areal radius $r(u, v)$ is related to the tortoise coordinate $r^{*}$ via

$$
r^{*} \equiv \frac{v-u}{2}=r(u, v)+a \log \left(\frac{r(u, v)}{a}-1\right) \simeq a+a \log \left(\frac{r(u, v)-a}{a}\right) .
$$

\footnotetext{
${ }^{7}$ Strictly speaking, $r-a \gtrsim N \ell_{p}^{2} / a$ is satisfied both outside and inside the apparent horizon. We refer to the region outside the Schwarzschild radius here.

${ }^{8}$ The choice of the domain $\left(\frac{N \ell_{p}^{2}}{2 a}, \frac{N \ell_{p}^{2}}{a}\right)$ is arbitrary, as long as it covers a neighbourhood of eq. A.1 where the Schwarzschild approximation is good.
} 
In the neighbourhood A.2, the Schwarzschild metric A.3 becomes approximately

$$
d s^{2} \simeq-C_{0}(u, v) d u d v+a^{2} d \Omega^{2}, \quad \text { where } \quad C_{0}(u, v) \equiv \frac{a}{r} e^{\frac{v-u-2 a}{2 a}} .
$$

In the neighbourhood $\mathrm{A} .2$, the metric A.3 means $C(u, v) \sim \mathcal{O}\left(\ell_{p}^{2} / a^{2}\right)$. Then, $\Sigma_{0}$, which is defined by eq. (3.8), is

$$
\Sigma_{0}(u, v) \simeq \frac{v-u}{2 a}-1+\log (a)
$$

\section{References}

[1] S. D. Mathur, "The Information paradox: A Pedagogical introduction," Class. Quant. Grav. 26, 224001 (2009) arXiv:0909.1038 [hep-th]].

[2] A. Almheiri, D. Marolf, J. Polchinski and J. Sully, "Black Holes: Complementarity or Firewalls?," JHEP 1302, 062 (2013) arXiv:1207.3123 [hep-th]];

[3] S. L. Braunstein, "Black hole entropy as entropy of entanglement, or it's curtains for the equivalence principle," [arXiv:0907.1190v1 [quant-ph]] published as S. L. Braunstein, S. Pirandola and K. Życzkowski, "Better Late than Never: Information Retrieval from Black Holes," Phys. Rev. Lett. 110, no. 10, 101301 (2013), for a similar prediction from different assumptions.

[4] P. C. W. Davies, S. A. Fulling and W. G. Unruh, "Energy-momentum Tensor Near an Evaporating Black Hole," Phys. Rev. D 13, 2720 (1976).

[5] S. M. Christensen and S. A. Fulling, "Trace Anomalies and the Hawking Effect," Phys. Rev. D 15, 2088 (1977).

[6] R. Brout, S. Massar, R. Parentani and P. Spindel, "A Primer for black hole quantum physics," Phys. Rept. 260, 329 (1995) arXiv:0710.4345 [gr-qc]].

[7] S. Massar, "The Semiclassical back reaction to black hole evaporation," Phys. Rev. D 52, 5857-5864 (1995). arXiv:gr-qc/9411039 [gr-qc]].

[8] P. M. Ho, Y. Matsuo and Y. Yokokura, "Analytic description of semiclassical black-hole geometry," Phys. Rev. D 102, no.2, 024090 (2020). arXiv:1912.12855 [hep-th]]. 
[9] V. Baccetti, R. B. Mann, S. Murk and D. R. Terno, "Energy-momentum tensor and metric near the Schwarzschild sphere," Phys. Rev. D 99, no.12, 124014 (2019) doi:10.1103/PhysRevD.99.124014 arXiv:1811.04495 [gr-qc]].

[10] V. Baccetti, S. Murk and D. R. Terno, "Black hole evaporation and semiclassical thin shell collapse," Phys. Rev. D 100, no.6, 064054 (2019) doi:10.1103/PhysRevD.100.064054 arXiv:1812.07727 [gr-qc]].

[11] I. Booth, "Black hole boundaries," Can. J. Phys. 83, 1073 (2005) gr-qc/0508107.

[12] J. Thornburg, "Event and apparent horizon finders for 3+1 numerical relativity," Living Rev. Rel. 10, 3 (2007) gr-qc/0512169.

[13] V. Faraoni, "Evolving black hole horizons in General Relativity and alternative gravity," Galaxies 1, no. 3, 114 (2013) arXiv:1309.4915 [gr-qc]].

[14] H. Kawai, Y. Matsuo and Y. Yokokura, "A Self-consistent Model of the Black Hole Evaporation," Int. J. Mod. Phys. A 28, 1350050 (2013) [arXiv:1302.4733 [hep-th]].

[15] H. Kawai and Y. Yokokura, "Phenomenological Description of the Interior of the Schwarzschild Black Hole," Int. J. Mod. Phys. A 30, 1550091 (2015) arXiv:1409.5784 [hep-th]]. P. M. Ho, "Comment on Self-Consistent Model of Black Hole Formation and Evaporation," JHEP 1508, 096 (2015) [arXiv:1505.02468 [hep-th]]. H. Kawai and Y. Yokokura, "Interior of Black Holes and Information Recovery," Phys. Rev. D 93, no. 4, 044011 (2016) arXiv:1509.08472 [hep-th]]. P. M. Ho, "The Absence of Horizon in Black-Hole Formation," Nucl. Phys. B 909, 394 (2016) arXiv:1510.07157 [hep-th]]. P. M. Ho, "Asymptotic Black Holes," Class. Quant. Grav. 34, no. 8, 085006 (2017) arXiv:1609.05775 [hep-th]]. H. Kawai and Y. Yokokura, "A Model of Black Hole Evaporation and 4D Weyl Anomaly," Universe 3, no. 2, 51 (2017) arXiv:1701.03455[hep-th]]. H. Kawai and Y. Yokokura, "Black Hole as a Quantum Field Configuration," Universe, 6(6), 77 (2020). arXiv:2002.10331 [hep-th]].

[16] V. P. Frolov and G. A. Vilkovisky, "Spherically Symmetric Collapse in Quantum Gravity," Phys. Lett. B 106, 307 (1981).

[17] T. A. Roman and P. G. Bergmann, "Stellar collapse without singularities?," Phys. Rev. D 28, 1265 (1983). 
[18] S. A. Hayward, "Formation and evaporation of regular black holes," Phys. Rev. Lett. 96, 031103 (2006) gr-qc/0506126.

[19] P. M. Ho and Y. Matsuo, JHEP 1906, 057 (2019) arXiv:1905.00898 [gr-qc]].

[20] P. Ho and Y. Yokokura, "Firewall From Effective Field Theory," arXiv:2004.04956 [hep-th]].

21

[21] P. M. Ho, "From Uneventful Horizon to Firewall in D-Dimensional Effective Theory," arXiv:2005.03817 [hep-th]]. 